\title{
Study on low-temperature crack resistance of zirconium tungstate modified asphalt mastic and asphalt mixtures
}

\author{
Z.S. Pei, J.Y. Yi* \& D.C. Feng \\ J.J. Li \\ China Railway Eryuan Engineering Group CO.LTD, Chengdu, China
}

School of Transportation Science and Engineering, Harbin Institute of Technology, Harbin, China

\begin{abstract}
During the service time of asphalt pavement, low-temperature cracking is one of the main deteriorations which could influence the performance of pavement and shorten the service life of the pavement. One of the main causes of low-temperature cracking is that the thermal stress inside the asphalt mixture cannot well relax along with time and then accumulate continuously until exceeding the tensile strength when the temperature drops suddenly in winter. To improve the thermal cracking resistance of asphalt mixture, zirconium tungstate (ZT) with a negative expansion property was introduced to partly replace the mineral powder in asphalt mortar and asphalt mixtures in this study. From the thermal contraction test, it was conducted that the ZT could reduce the low-temperature contraction coefficient of asphalt mortar as well as asphalt mixture. Besides, the contraction coefficient of mortar had a good positive correlation with the contraction coefficient of mixtures under different ZT content conditions. Results of the Thermal Stress Restrained Specimen Test showed that the cracking temperature and transition temperature of asphalt mixtures reduced with the increase of ZT contents. The research of this subject can provide a new idea for solving the low-temperature contraction cracking and design of asphalt pavement.
\end{abstract}

Keywords: Asphalt mortar, asphalt mixtures, zirconium tungstate, low-temperature cracking

\section{INTRODUCTION}

In the course of pavement service, low-temperature cracking is one of the main failure modes, which has appeared in many countries in the world (Das et al. 2013, Son et al. 2019). Because most of the materials have the characteristics of thermal expansion and cold contraction, it is difficult to avoid temperature shrinkage cracks in the pavement under extreme temperature (Park et al. 2016, Judycki, 2016). For example, in China, the technical index of asphalt pavement design specification for the high-grade highway is that no more than three low-temperature cracks can be allowed within 100 meters after completion acceptance. However, low-temperature cracking not only affects the driving comfort of the road surface but also has a greater impact on the continuity and safety of the road surface, which will increase the fuel cost and time cost of driving (Aflaki and Hajikarimi 2012, Sun et al. 2018). Besides, water and impurities could corrode the pavement structure through 
cracks, and the water remaining in the pores will cause further damage to the pavement structure when it is frozen at low temperatures (Ren and Sun 2017, Ahmad and Khawaja 2018).

To improve the ability of pavement structure to resist low-temperature cracking, scholars have carried out a lot of related research. Results indicate that the asphalt binder has a significant influence on the low-temperature performance of asphalt mixture (Judycki, 2014). However, asphalt binders show "hard" and "brittle" mechanical properties in the low-temperature environment. Besides, with the aging process, the asphalt becomes more "hard" and "brittle", which brings adverse impact on the low-temperature performance of asphalt mixture (Wu et al. 2012, Wang et al. 2013). Therefore, many researches focus on improving the performance of asphalt binders to improve the ability of asphalt mixture to resist low temperature cracking. The modification of asphalt has aroused great interest of researchers. The results show that styrene-butadiene-styrene (SBS) or rubber powder can improve the low-temperature performance of asphalt binder and reduce the low-temperature cracking of the asphalt mixture (Nam and Bahia 2009, Hajikarimi et al. 2013). Besides, the addition of $0.2 \sim 0.4 \%$ lignin fiber can also improve the low-temperature performance of asphalt mixture (Luo et al. 2019, Yue et al. 2019). The thermal conductivity of the asphalt mixture can be reduced by adding expanded polypropylene (EPP) microspheres to the asphalt mixture, to change its thermodynamic performance (Shi et al. 2019). Besides, the use of lightweight aggregate can reduce the thermal conductivity of asphalt mixture, to improve its mechanical properties at low temperatures (Khan and Mrawira 2008). Open-graded friction course (OGFC) has a lower thermal shrinkage coefficient and is more potential to resist low temperature cracking than asphalt concrete (AC) (Islam et al. 2018). Although researchers have carried out a lot of research on asphalt binder, aggregate, and mixture gradation to improve the lowtemperature cracking performance of asphalt mixture, the effect is still not particularly obvious. The main reason is that the contraction stress of the asphalt mixture in the lowtemperature environment is difficult to avoid. If the thermal stress in the low-temperature environment can be greatly reduced, the low-temperature cracking resistance of asphalt mixture will be greatly improved. Negative thermal expansion materials can be tried to achieve this function.

Zirconium tungstate (ZT) was successfully prepared in 1996 by Mary et al (Mary et al. 1996). They found that the negative thermal expansion temperature range of zirconium tungstate is $-273{ }^{\circ} \mathrm{C} \sim 777^{\circ} \mathrm{C}$, and the isotropic properties of ZT was good. This discovery makes it possible for the practical application of negative thermal expansion materials. ZT has been widely used in the preparation of composite materials due to its wide range of reverse expansion temperature range and isotropy. ZT was combined with aluminum alloy to make the alloy. When the volume of ZT was three times that of aluminum alloy, the contraction coefficient of the alloy could reach 0 (Matsumoto et al. 2003). ZT and zirconia were used to produce composite materials, and the expansion coefficient of the composites was close to 0 when the mass of zirconia was twice that of ZT (Niwa et al. 2004). Also, ZT was used to improve the performance of cement concrete, and the results show that the contraction coefficient of cement concrete was close to 0 when the mass fraction of ZT reaches $60 \%$ (Kofteros et al. 2001). If the thermal contraction coefficient of asphalt mixture can be improved by using ZT, it will greatly change the anti-cracking ability of asphalt mixture at low temperatures.

Based on the above analysis, ZT with a negative expansion property was introduced to partly replace the mineral powder in asphalt mastic and asphalt mixtures in this study. Through the performance changes of asphalt mortar and asphalt mixture, the influence of zirconium tungstate on the low-temperature crack resistance of asphalt mixture was explored and evaluated. The research of this subject can provide a new idea for solving the lowtemperature contraction cracking and design of asphalt pavement. 


\section{MATERIALS}

\subsection{Asphalt}

The 70 base asphalt selected in this project is the asphalt binder commonly used in northern China, and its technical indicators are shown in Table 1. The test method refers to "Standard test methods of bitumen and bituminous mixtures for highway engineering" (JTG E20-2011).

Table 1. Technical index of 70 base asphalt.

\begin{tabular}{llll}
\hline Technical indexes & Test result & Criteria & Testing method \\
\hline Penetration $\left(25^{\circ} \mathrm{C}, 100 \mathrm{~g}, 5 \mathrm{~s}\right) / 0.1 \mathrm{~mm}$ & 69 & $60-80$ & \\
Penetration index & -0.15 & $1.5 \sim+1.0$ & JTG T0604-2011 \\
Extension $\left(10^{\circ} \mathrm{C}\right) /(\mathrm{cm})$ & 31 & $\geq 15$ & \\
Extension $\left(15^{\circ} \mathrm{C}\right) /(\mathrm{cm})$ & $>100$ & $\geq 100$ & JTG T0605-2011 \\
Softening point $(\mathrm{R} \& \mathrm{~B}) /{ }^{\circ} \mathrm{C}$ & 51 & $\geq 45$ & $\mathrm{JTG} \mathrm{T0606-2011}$ \\
Density $\left(15^{\circ} \mathrm{C}\right) / \mathrm{kg} / \mathrm{m}^{3}$ & 1.003 & - & $\mathrm{JTG}$ T0603-2011 \\
Residue after RTFOT & & & \\
Quality change $/ \%$ & 0.53 & \pm 0.8 & JTG T0610-2011 \\
Residual penetration ratio $\left(25^{\circ} \mathrm{C}\right) / \%$ & 63 & $\geq 61$ & JTG T0604-2011 \\
Extension $\left(10^{\circ} \mathrm{C}\right) /(\mathrm{cm})$ & 11 & $\geq 6$ & JTG T0605-2011 \\
\hline
\end{tabular}

\subsection{Fillers}

ZT is introduced to partly replace the mineral powder in asphalt mastic and asphalt mixtures. The appearance of ZT used in this study is a bright green powder, and the purity is above $99.5 \%$. The mineral powder is made of limestone, and its technical indicators are shown in Table 2, and the test method refers to "Test methods of aggregate for highway engineering" (JTG E42-2005). In this study, the fillers are divided into five kinds, and the mass ratio of ZT to the total filler is $0 \%, 20 \%, 40 \%, 60 \%$, and $80 \%$, respectively.

Table 2. Technical index of mineral powder.

\begin{tabular}{llll}
\hline Technical indexes & Test result & Criteria & Testing method \\
\hline Density $/ \mathrm{kg} / \mathrm{m}^{3}$ & 2.63 & $\geq 2.50$ & JTG E42-T0352-2005 \\
Moisture content $/ \%$ & 0.61 & $\leq 1$ & JTG E42-T0103-2005 \\
Hydrophilic coefficient & 0.6 & $<1$ & JTG E42-T0353-2005 \\
Appearance & No agglomeration & No agglomeration & - \\
\hline
\end{tabular}

\subsection{Asphalt concerete}

The asphalt concrete (AC-13) is used as the test object, and the aggregate and gradation are carried out according to the requirements of "Technical specification for construction of highway asphalt pavement" (JTG F40-2004). The optimum asphalt content is determined to be $4.9 \%$ through the mixture proportion design. Besides, the mixing ratio of asphalt mortar is selected as the mass ratio of asphalt and filler in AC-13. 


\section{TEST METHODS}

\subsection{Thermal contraction test of asphalt mortar}

The coefficient of thermal contraction of asphalt mortar refers to the volume change value with the change of unit temperature, which could well characterize the low-temperature performance of asphalt mortar (Islam and Tarefder 2015). The low-temperature performance of asphalt mortar deteriorates with the increase of temperature contraction coefficient.

To measure the volume deformation of the mortar more accurately, the contraction coefficient test device developed by the research group is used to carry out the test, as shown in Figure 1. During the test, the prepared asphalt mortar specimen is put into the metal chamber of the equipment, and then the top cover is covered. The fixed bolts are tightened, and the base is adjusted to make the measuring instrument in a horizontal state. Then ethanol is injected into the glass calibration tube, and the device is placed in the environment of different temperatures. Finally, the change value of the mortar volume is calculated according to the change of ethanol liquid scale.

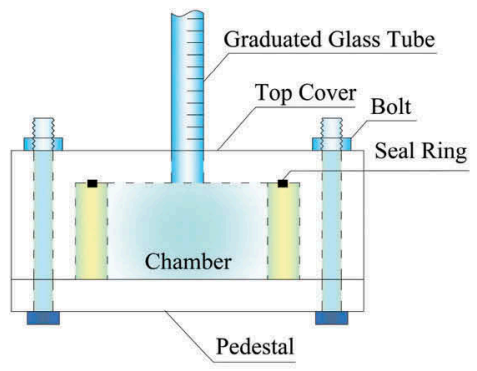

(a) Side view

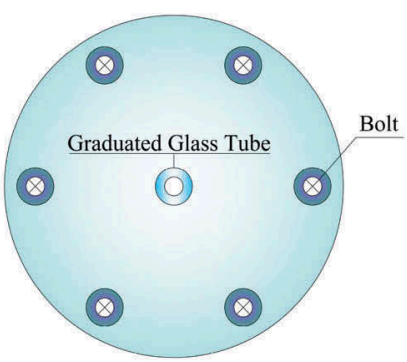

(b) Top view

Figure 1. Device of asphalt mortar contraction coefficient.

During the test, constant temperature equipment is used for temperature control. Firstly, the initial temperature is set, and the test device is put into the temperature control equipment for 4 hours to read the initial value of the calibration tube. Then the temperature is adjusted to the next setting value and the value of the calibration tube is read after 4 hours of heat preservation. In this way, the volume changes of asphalt mortar at $-20^{\circ} \mathrm{C},-10{ }^{\circ} \mathrm{C}$, and $0{ }^{\circ} \mathrm{C}$ are measured. In the process of temperature change, the volume of the test device and ethanol will change as well, resulting in system errors. Therefore, the metal specimen with a known volume contraction coefficient and the same volume as the standard asphalt mortar specimen is used to calibrate the system errors. The calibration process is also carried out at $-20^{\circ} \mathrm{C},-10$ ${ }^{\circ} \mathrm{C}$, and $0{ }^{\circ} \mathrm{C}$. Finally, the thermal contraction coefficient of asphalt mortar is calculated according to formula (1).

$$
\beta_{i}=\frac{\left(L_{i+1}-L_{i}\right)-\Delta V_{\text {system-errors }}}{\left(T_{i}-T_{i+1}\right) \times V_{\text {specimen }}}
$$

where $\beta_{i}$ is the coefficient of thermal contraction of asphalt mortar, $L_{i}$ is the value of calibration tube at time $T_{i}, \Delta V_{\text {system errors }}$ is the system errors calibrated by the metal specimen, $\Delta V_{\text {specimen }}$ is the volume of asphalt mortar specimen. 


\subsection{Thermal contraction test of asphalt mixture}

The contraction capacity of asphalt mixture can well reflect its low-temperature performance. Many parameters affect the thermal contraction coefficient of asphalt mixture, including mineral aggregate gradation of asphalt mixture, asphalt properties, and asphalt dosage. The thermal contraction coefficient of the asphalt mixture is about $2.21 \times 10^{-5} \sim 3.33 \times 10^{-5}\left(/{ }^{\circ} \mathrm{C}\right)$ in several studies (Zeng and Shields 1999, Akentuna et al. 2018). To verify the low-temperature performance of ZT modified asphalt mixture, a test on the thermal contraction coefficient of the asphalt mixture is carried out.

The test system includes an incubator for temperature control, a test chamber for holding test pieces, a displacement sensor system, and a temperature sensor system. The asphalt concrete specimens are formed by the method of the wheel rolling and cut into $200 \mathrm{~mm} \times 35 \mathrm{~mm}$ $\times 30 \mathrm{~mm}$ prisms.

The initial temperature was set at $20^{\circ} \mathrm{C}$ with a cooling rate of $5{ }^{\circ} \mathrm{C} / \mathrm{h}$. The temperature range is set at $-20{ }^{\circ} \mathrm{C} \sim 20^{\circ} \mathrm{C}$. Because the measurement system will shrink due to the change of temperature, the instrument without the test piece is calibrated, and the calibration temperature range is $-20^{\circ} \mathrm{C} \sim 20^{\circ} \mathrm{C}$. Finally, the thermal contraction coefficient of the asphalt mixture is calculated according to formula (2).

$$
\alpha=\frac{\left(L_{i+1}-L_{i}\right)-L_{\text {system-errors }}}{L_{\text {specimen }} \times\left(T_{m+1}-T_{m}\right)}
$$

where $\alpha$ is the thermal contraction coefficient of asphalt mixture, $L_{i}$ is the value of displacement sensor at time $T_{i}(\mathrm{~mm}), L_{\text {system }}$ errors is the system errors calibrated by standard test, $L_{\text {specimen }}$ is the height of test piece ( $\left.\mathrm{mm}\right)$.

\subsection{The thermal stress restrained specimen test}

The device of thermal stress restrained specimen test is composed of a temperature control system, loading system, data acquisition system, and measurement system, as shown in Figure 2 . The device can accurately control the length variation of the specimen with an accuracy of $3 \mu \mathrm{m}$. The maximum allowable deformation of asphalt mixture is $4 \mathrm{~cm}$. The temperature sensor is used to measure the test temperature, and the accuracy is $0.25{ }^{\circ} \mathrm{C}$. The accuracy of the stress measuring device is $10 \mathrm{~N}$.

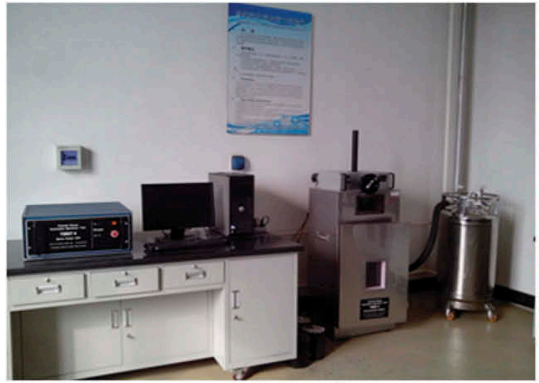

(a) Device of test

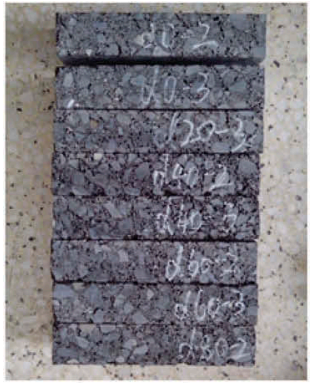

(b) Specimen

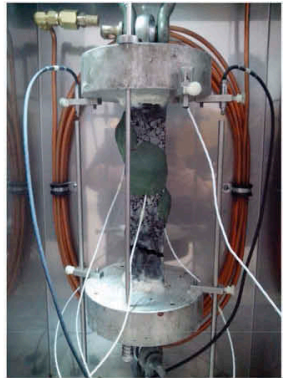

(c) Test process

Figure 2. Test of thermal stress restrained specimen. 


\section{RESULTS AND DISCUSSIONS}

\subsection{Coefficient of thermal contraction asphalt mortar}

The contraction coefficient of asphalt mortar containing $0 \%, 20 \%, 40 \%, 60 \%$, and $80 \% \mathrm{ZT}$ was tested. At least 3 parallel tests were conducted for each group. Finally, the contraction coefficient of asphalt mortar is obtained, as shown in Table 3.

It can be seen from Table 3 that the thermal contraction coefficient of asphalt mortar is not completely the same in different temperature ranges. However, with the addition of ZT, the thermal contraction coefficient of asphalt mortar decreases gradually. The results show that the negative expansion of zirconium tungstate can reduce the thermal contraction coefficient of asphalt mortar. The average contraction coefficient of $80 \%$ group is $60.55 \%$ lower than that of the $0 \%$ group. The addition of zirconium tungstate can effectively slow down the contraction trend of asphalt mortar in the low-temperature environment.

Table 3. Test results of volume contraction of asphalt mortar.

\begin{tabular}{|c|c|c|c|c|c|}
\hline \multirow{2}{*}{$\begin{array}{l}\text { Content of } \\
\mathrm{ZT} \text { in filler } \\
0 \%\end{array}$} & \multirow{2}{*}{$\begin{array}{l}T_{i}\left({ }^{\circ} \mathrm{C}\right) \\
0\end{array}$} & \multicolumn{3}{|c|}{ Contraction coefficient $\left(/{ }^{\circ} \mathrm{C} \times 10^{-4}\right)$} & \multirow{2}{*}{$\begin{array}{l}\begin{array}{l}\text { Average contraction } \\
\text { coefficient }\left(/{ }^{\circ} \mathrm{C} \times 10^{-4}\right)\end{array} \\
6.92\end{array}$} \\
\hline & & 7.24 & 8.44 & 7.52 & \\
\hline & $\begin{array}{l}-10 \\
-20\end{array}$ & 5.89 & 5.65 & 6.75 & \\
\hline \multirow[t]{2}{*}{$20 \%$} & 0 & 6.69 & 5.94 & 6.95 & 6.12 \\
\hline & $\begin{array}{l}-10 \\
-20\end{array}$ & 6.03 & 5.62 & 5.46 & \\
\hline \multirow[t]{2}{*}{$40 \%$} & 0 & 6.77 & 5.96 & 5.68 & 5.32 \\
\hline & $\begin{array}{l}-10 \\
-20\end{array}$ & 4.36 & 4.87 & 4.26 & \\
\hline \multirow[t]{3}{*}{$60 \%$} & 0 & 3.48 & 4.1 & 3.67 & 4.33 \\
\hline & -10 & 5.2 & 4.44 & 5.06 & \\
\hline & -20 & & & & \\
\hline \multirow{3}{*}{$80 \%$} & 0 & 2.29 & 1.98 & 2.19 & 2.73 \\
\hline & -10 & 3.18 & 3.2 & 3.54 & \\
\hline & -20 & & & & \\
\hline
\end{tabular}

\subsection{Coefficient of thermal contraction asphalt mixture}

The displacement variation under unit temperature is taken as the index of the contraction coefficient of the asphalt mixture. In the experiment, the cooling rate was $5^{\circ} \mathrm{C} / \mathrm{h}$, and the data were collected every 1 hour. The contraction of asphalt mixture with different content of ZT is obtained, as shown in Figure 3. According to formula (2), the thermal contraction coefficient of the asphalt mixture is calculated, as shown in Table 4.

Table 4. Thermal contraction coefficient of asphalt mixture.

\begin{tabular}{lll}
\hline Content of ZT in filler & Contraction coefficient $\left(\times 10^{-5 / 0} \mathrm{C}\right)$ & Correlation coefficient \\
\hline $0 \%$ & 3.34 & 0.9884 \\
$20 \%$ & 3.08 & 0.9817 \\
$40 \%$ & 2.83 & 0.9645 \\
$60 \%$ & 2.78 & 0.9598 \\
$80 \%$ & 2.51 & 0.9489 \\
\hline
\end{tabular}


The contraction coefficient of asphalt mixture ( $0 \%$ group) without ZT is $3.34 \times 10-5\left(/{ }^{\circ} \mathrm{C}\right)$ in the range from $-20^{\circ} \mathrm{C}$ to $20^{\circ} \mathrm{C}$, which is very close to the contraction coefficient of asphalt mixture measured by other scholars (Dong et al. 2012, Akentuna et al. 2018). It can be seen from Table 4 that the contraction coefficient of the asphalt mixture gradually decreases with the increase of ZT content. The contraction coefficient of $80 \%$ group was $24.85 \%$ lower than that of the $0 \%$ group. Compared with the contraction coefficient test of asphalt mortar, the decrease range of the contraction coefficient of the asphalt mixture with ZT is smaller. The main reason is that the stone accounts for the majority of the asphalt mixture, and the contraction coefficient of the mixture is affected by the properties of the stone. On the whole, the low-temperature contraction trend of asphalt mixture is weakened by adding ZT.

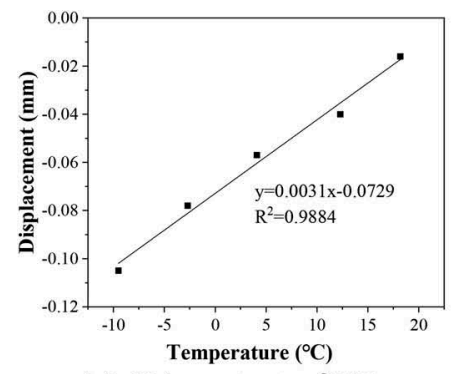

(a) $0 \%$ content of ZT

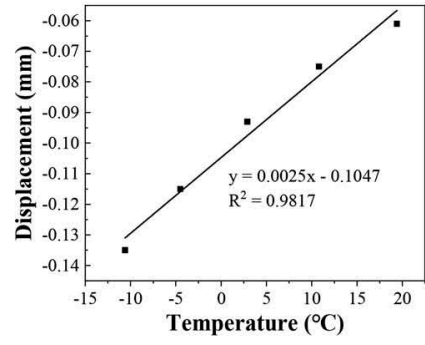

(b) $20 \%$ content of ZT

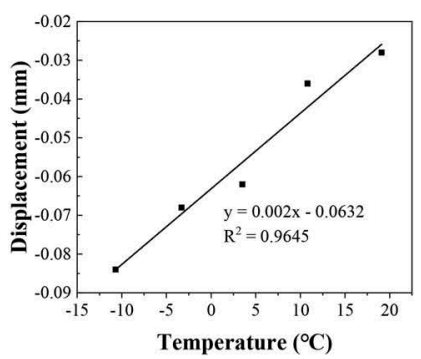

(c) $40 \%$ content of ZT

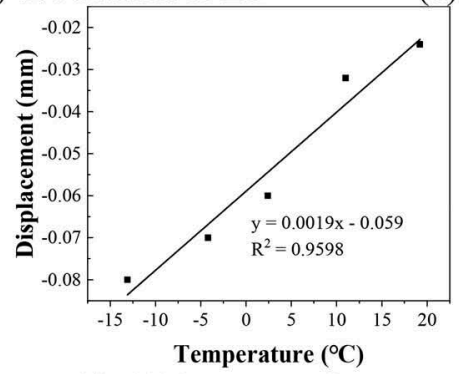

(d) $60 \%$ content of ZT

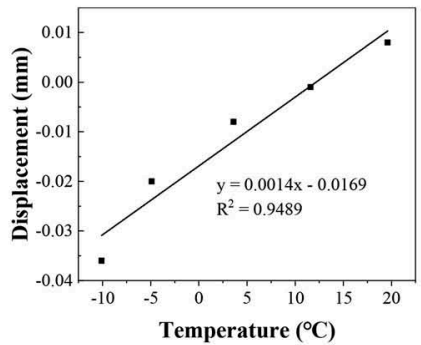

(e) $80 \%$ content of ZT

Figure 3. Fitting results of contraction test of asphalt mixture.

Asphalt mixture is composed of asphalt mortar and stone, and there is a close connection on low-temperature performance between asphalt mortar and asphalt mixture. The correlation of contraction coefficient between ZT modified asphalt mortar and asphalt mixture was analyzed. The contraction coefficient of ZT modified asphalt mixture decreases with the decrease of contraction coefficient of the asphalt mortar, and the correlation coefficient reaches to 0.9365 , as shown in Figure 4 . It shows that the contraction coefficient of the mixture has a good positive correlation with the contraction coefficient of mortar.

\subsection{Rusults of the thermal stress restrained specimen test}

The thermal stress restrained specimen test can simulate the stress process of pavement cracking at low temperatures. Among many evaluation indexes, the cracking temperature and the transition temperature have good consistency in evaluating the low-temperature performance of the asphalt mixture. In this study, the low-temperature performance of ZT modified asphalt mixture is evaluated by cracking temperature and transition temperature. The results of the thermal stress restrained specimen test are shown in Figure 5. 


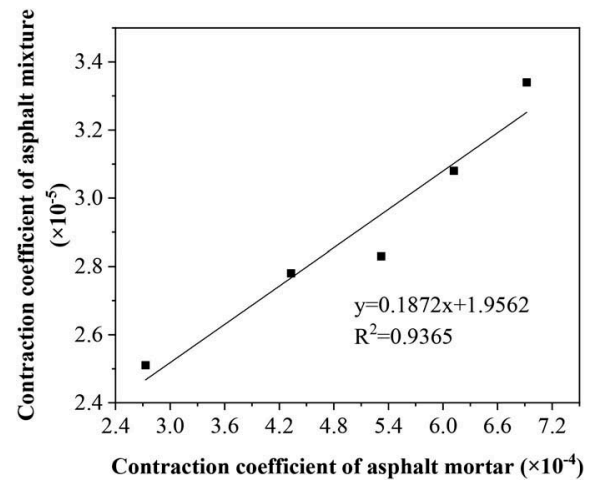

Figure 4. Fitting results of contraction coefficient of asphalt mortar and asphalt mixture.

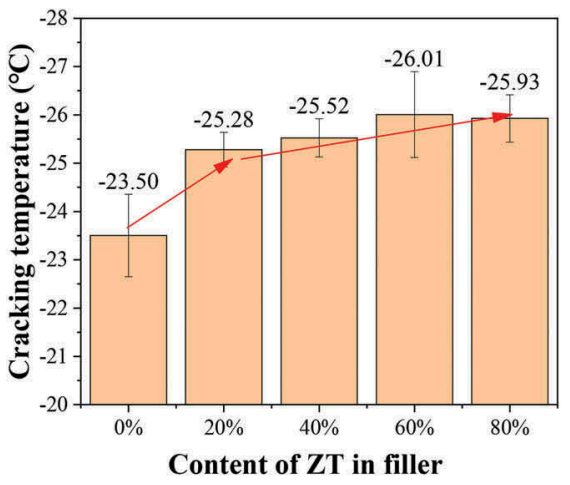

(a) Cracking temperature of asphalt mixture

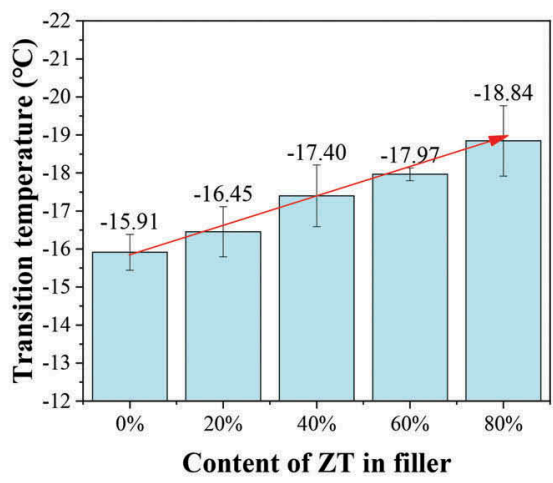

(b) Transition temperature of asphalt mixture

Figure 5. Results of thermal stress restrained specimen test.

It can be seen from Figure 5 that the cracking temperature and transition temperature of asphalt mixture gradually decrease with the increase of ZT content, which shows that the low-temperature performance of asphalt mixture could be improved by adding ZT. And the cracking temperature of the test group added ZT is at least $1.8^{\circ} \mathrm{C}$ lower than that of the ordinary asphalt mixture. Besides, the cracking temperature of the $80 \%$ group was $10.34 \%$ lower than that of the $0 \%$ group, and the transition temperature of the $80 \%$ group was $15.27 \%$ lower than that of the $0 \%$ group. It shows that the low-temperature performance of the ZT-modified asphalt mixture is better than that of ordinary asphalt mixture. Moreover, the cracking temperature and transition temperature of the asphalt mixture have a good consistency.

Although the cracking temperature of asphalt mixture only decreases by $2-3{ }^{\circ} \mathrm{C}$ after adding $\mathrm{ZT}$, the thermal stress restrained specimen test is also related to the size effect of the specimen, which can not fully represent the real service condition of asphalt pavement. Therefore, the test results mainly show the influence trend of ZT addition on the low-temperature performance of asphalt mixture and provide some new ideas to improve the low-temperature performance. 


\section{CONCLUSION}

(1) The negative expansion of ZT can reduce the thermal contraction coefficient of asphalt mortar. And the average contraction coefficient of asphalt mortar of $80 \%$ group is $60.55 \%$ lower than that of $0 \%$ group.

(2) The contraction coefficient of asphalt mixture gradually decreases with the increase of ZT content. And the contraction coefficient of the $80 \%$ group was $24.85 \%$ lower than that of the $0 \%$ group. Besides, the contraction coefficient of the asphalt mixture has a good positive correlation with the contraction coefficient of the asphalt mortar.

(3) The low-temperature performance of ZT modified asphalt mixture is better than that of ordinary asphalt mixture. The cracking temperature of the asphalt mixture of $80 \%$ group is $10.34 \%$ lower than that of $0 \%$ group, and the transition temperature of the asphalt mixture of $80 \%$ group is $15.27 \%$ lower than that of $0 \%$ group.

(4) The addition of ZT could improve the cracking resistance of asphalt mixture at low temperatures. Although the cost of ZT is high at present, the unit price of material has been greatly reduced with the improvement of preparation technology in recent years. In the following study, other mechanical properties of asphalt mixtures mixed with ZT will be fully verified. We hope that this research can provide a new idea for solving the lowtemperature shrinkage cracking and design of asphalt pavement.

\section{REFERENCES}

Aflaki, S. and Hajikarimi, P., 2012. Construction and Building Materials. Implementing viscoelastic rheological methods to evaluate low temperature performance of modified asphalt binders, 36, $110-118$.

Ahmad, T. and Khawaja, H., 2018. International Journal of Multiphysics. Review of Low-Temperature Crack (LTC) Developments in Asphalt Pavements, 12 (2), 169-187.

Akentuna, M., Kim, S. S., Nazzal, M., et al., 2017. Journal of Materials in Civil Engineering. Asphalt Mixture CTE Measurement and the Determination of Factors Affecting CTE, 29 (6), 04017010.

Das, P. K., Jelagin, D., Birgisson, B., 2013. Construction and Building Materials. Evaluation of the low temperature cracking performance of asphalt mixtures utilizing HMA fracture mechanics, 47, 594-600.

Dong, D., Huang, X., Li, X., et al., 2012. Construction and Building Materials. Swelling process of rubber in asphalt and its effect on the structure and properties of rubber and asphalt, 29, 316-322.

Hajikarimi, P., Aflaki, S., Hoseini, A. S., 2013. Construction and Building Materials. Implementing fractional viscoelastic model to evaluate low temperature characteristics of crumb rubber and gilsonite modified asphalt binders, 49, 682-687.

Islam, M. R., Rahman, A. S. M. A., Tarefder, R. A., 2018. Journal of Cold Regions Engineering. Open Graded Friction Course in Resisting Low-Temperature Transverse Cracking in Asphalt Pavement, 32 (2), 04018006.

Islam, M. R. and Tarefder, R. A., 2015. Journal of Materials in Civil Engineering. Coefficients of Thermal Contraction and Expansion of Asphalt Concrete in the Laboratory, 27 (11), 04015020.

Judycki, J., 2016. International Journal of Pavement Engineering. A new viscoelastic method of calculation of low-temperature thermal stresses in asphalt layers of pavements, 19 (1), 24-36.

Judycki, J., 2014. Construction and Building Materials. Influence of low-temperature physical hardening on stiffness and tensile strength of asphalt concrete and stone mastic asphalt, 61, 191-199.

Khan, A. and Mrawira, D., 2008. Journal of Testing and Evaluation. Influence of Selected Mix Design Factors on the Thermal Behavior of Lightweight Aggregate Asphalt Mixes, 36 (6), 492-499.

Kofteros, M., Rodriguez, S., Tandon, V., et al., 2001. Scripta Materialia. A preliminary study of thermal expansion compensation in cement by ZrW2O8 additions, 45 (4), 369-374.

Luo, D., Khater, A., Yue, Y. C., et al., 2019. Construction and Building Materials. The performance of asphalt mixtures modified with lignin fiber and glass fiber: A review, 209, 377-387.

Mary, T. A., Evans, J. S. O., Vogt, T., et al., 1996. SCIENCE. Negative thermal expansion from 0.3 to 1050 Kelvin in ZrW2O8, 272 (5258), 90-92.

Matsumoto, A., Kobayashi, K., Nishio, T., et al., 2003. Materials Science Forum. Fabrication and Thermal Expansion of Al-ZrW2O8 Composites by Pulse Current Sintering Process, 426-432 (3), $2279-2284$. 
Nam, K. and Bahia, H. U., 2009. Journal of Materials in Civil Engineering. Effect of Modification on Fracture Failure and Thermal-Volumetric Properties of Asphalt Binders, 21 (5), 198-209.

Niwa, E., Wakamiko, S., Ichikawa, T., et al., 2004. Journal of the Ceramic Society of Japan. Preparation of Dense $\mathrm{ZrO} / \mathrm{ZrW} 2 \mathrm{O} 8$ Cosintered Ceramics with Controlled Thermal Expansion Coefficients, 112 (1305), 271-275.

Park J. W., et al., 2016. Polymer Testing. Characteristic shrinkage evaluation of photocurable materials, $56,344-353$.

Ren, J. L. and Sun, L., 2017. Engineering Fracture Mechanics. Characterizing Air Void Effect on Fracture of Asphalt Concrete at Low-temperature using Discrete Element Method, 170, $23-43$.

Shi, X. J., Rew, Y., Ivers, E., et al., 2019. International Journal of Pavement Engineering. Effects of thermally modified asphalt concrete on pavement temperature, 20 (6), 669-681.

Son S., Said I. M., Al-Qadi, I. L., 2019. Construction and Building Materials. Fracture properties of asphalt concrete under various displacement conditions and temperatures, 222, 332-341.

Sun, Z. Q., Xu, H. N., Tan, Y. Q., et al., 2019. Construction and Building Materials. Low-temperature performance of asphalt mixture based on statistical analysis of winter temperature extremes: A case study of Harbin China, 208, 258-268.

Wang, D., Linbing, W., Christian, D., et al., 2013. Journal of Materials in Civil Engineering. Fatigue Properties of Asphalt Materials at Low In-Service Temperatures, 25 (9), 1220-1227.

Wu, S. P., Zhu, G. J., Pang, L., et al., 2008. Key Engineering Materials. Influences of Aging History on Low Temperature Performance of Asphalt Concrete, 385-387, 493-496.

Yue, Y. C., Abdelsalam, M., Luo, D., et al., 2019. Materials. Evaluation of the Properties of Asphalt Mixes Modified with Diatomite and Lignin Fiber: A Review, 12 (3), 400.

Zeng, M. L. and Shields, D. H., 1999. Canadian Journal of Civil Engineering. Nonlinear thermal expansion and contraction of asphalt concrete, 26 (1), 26-34. 